\title{
Visual Vehicle Tracking Using Direction-based Particle Filter with Online Threshold Adaptation
}

\author{
Mustafa Eren Yıldırım, Burak Merdenyan, Yucel Batu Salman
}

\begin{abstract}
In various applications of computer vision, nonlinearity and non-Gaussianity must be considered to have an accurate and realistic modeling. In this sense, particle filter is preferred for tracking. In this paper, a modified particle filter which takes the direction of the vehicle into account and distributes the particles according an automatic thresholding scheme is used. After obtaining the vehicle direction, particles are weighted according to their angular similarities to the vehicle. Particles having angular distance greater than a threshold are eliminated. Thus, the remained particles that are moving in same or similar direction with vehicle, increase their own probability of likelihood. This threshold is decided online while the algorithm runs. Depending on the number of particles used, this scheme increases or decreases the threshold. This scheme prevents the system from failures due to insufficient number of particles and also from using excessive number of particles. Proposed algorithm is compared with condensation algorithm and Camshift algorithm. According to the results, proposed algorithm surpasses the other algorithms in terms of tracking period and precision.
\end{abstract}

Keywords-automatic thresholding, direction-based particle filter, vehicle tracking

\section{Introduction}

Object tracking has become a significant challenge in computer vision for various applications such as robotics [1], surveillance systems [2], medical imaging and humancomputer interaction [3]. The primary objective of tracking is estimating the states of the target object in subsequent frames accurately. There are three primary approaches of object tracking; video-based tracking [4], remote-sensing based tracking [5], and lidar sensing [6]. However, the video-based tracking algorithms are preferred to the others since such approaches have the capability of estimating the relative positions and classification of surrounding vehicles very accurately even in remote environments $[7,8]$.

Video-based vehicle tracking algorithms are gathered a great interest by the researchers with the new advances in object-detection techniques, high computational devices, multisensory systems and high definition cameras [2, 1, 9]. In this sense, several vehicle tracking algorithms have been introduced in using a wide range of approaches [10-14].

M.Eren Yıldırım, Y.Batu Salman

Bahcesehir University

Turkey

Burak Merdenyan

University of York

United Kingdom
Yet it is still a difficult task because of the deformation, illumination, background clutters and collision of the recorded video [15]. There exists two distinguished approaches in conventional video-based vehicle tracking: the one considering identification of vehicle in a frame and the estimation of object correspondence in successive frames independently [16] and the other operates both issues together and jointly [17].

Particle filter is a sampling-based tracking method which has the capability of solving nonlinear and non-Gaussian challenges in a range of applications [18]. It is basically a Bayesian filtering technique using the Sequential Mote Carlo method to estimate the system state and uses a sequential importance sampling approach to generate updated particle set [19]. The main drawback of particle filter algorithm is its need of a large number of particles to estimate the state of target correctly specifically when occlusion occur. This will certainly increase the computational complexity. It is expected to establish a particle filter algorithm which is able to perform accurately with the minimum number of particles. Therefore, a robust tracking performance achievement still remains a significant research.

An iterative particle filter algorithm was presented which is able to increase the accuracy but could not reduce the computational burden [20]. In [21], authors proposed a multifeature target representation with particle filter, although, it has shown success in accuracy, the computational load remained high. A layered particle filter technique which combines probabilistic and deterministic methods to overcome well-known barriers in multiple tracking processes was proposed [13]. Although better posterior probability density representation was presented, some errors were recorded under occlusion. A top-down visual attention computational model was presented which is based on frequency analysis and incorporates it to particle filter [22]. Another method with adaptive region-wise linear subspace expression with an iterative particle filter was introduced, however could not handle situations of complete occlusion [23]. Color histogram based tracking was generated as a robust one against noise and occlusion but failed with the existence of illumination changes [24]. Perez et al. [25] integrated a color histogram in a particle filter. In order to advance proposal distribution of particle filter, an advanced Kalman method was also introduced [26]. Yildirim et al. [14] introduced a particle filter method that distributes and filters particles according to their angular differences to the target vehicles' motion. This model reduced the particle consumption while increasing the tracking accuracy and performing robust against noise.

In this study, a modified particle filter algorithm is presented which distributes the particles depending on an automatic thresholding scheme. Particles are weighted based 
on their angular similarities to the target vehicle after the vehicle direction is initially obtained. Particles only having the same and similar motion direction with the vehicle are kept to increase their own probability of likelihood. The online threshold is valued according to the number of particles used. Using excessive or insufficient number of particles is prevented in this model. The experimental outcome of our novel approach is compared with camshift and condensation algorithms. Findings have shown that the proposed algorithm generated better results in terms of tracking time and precision.

\section{Direction-based Particle Filter}

Particle filter is a technique for implementing a recursive Bayesian filter by Monte Carlo method. The key idea of particle filter is to represent the posterior density function by a set of random particles with their corresponding weights and estimate state parameters based on these particles and weights. Weight of each particle changes depending on the observation of current frame. Main steps of particle filter are;

- Initialization of particle probability

- Propagation of particles

- Update of observation model.

- Normalization of particles.

- Estimation of target state parameters.

- Resampling of particles.

In our paper [14], we have presented a direction-based particle filter algorithm for vehicle tracking. The basis of it was Condensation algorithm[28]. Direction of the vehicle was obtained by (1);

$$
\theta_{t-1}=\tan ^{-1}\left(\frac{\Delta y}{\Delta x}\right)=\tan ^{-1}\left(\frac{y_{t}-y_{t-1}}{x_{t}-x_{t-1}}\right)
$$

in which $\left(x_{t}, y_{t}\right)$ and $\left(x_{t-1}, y_{t-1}\right)$ refer to center points of the vehicle in current and previous frames, $t$ is time index. This direction is updated periodically by using approximate median method [29].

The angle between the vehicle and each particle is calculated to identify the angular distance of each particle to the direction of motion of the vehicle. This distance is used to weight the particles and update their distribution around the vehicle. This calculation can be performed by using the distributed particles of the current time step and the vehicle's estimated location in the previous time step. Angle of a particular particle with reference to the target's position in the previous frame is calculated in (2). $N$ is the number of particles.

$$
\beta_{t-1}^{(n)}=\tan ^{-1}\left(\frac{\Delta y}{\Delta x}\right)=\tan ^{-1}\left(\frac{y_{t-1}-y_{t}^{(n)}}{x_{t-1}-x_{t}^{(n)}}\right) \quad n=1,2, \ldots, N
$$

The difference between the direction of motion and each value of $\beta_{t-1}^{(n)}$ is obtained by (3).

$$
\psi_{t-1}^{(n)}=\left|\theta_{t-1}-\beta_{t-1}^{(n)}\right| \quad n=1,2, \ldots, N
$$

Filtering of particles is done by applying a threshold control to all the results of (3) as shown in (4).

$$
\pi_{t}^{(n)}=\left(\begin{array}{ccc}
0 & \text { if } & \psi_{t-1}^{(n)} \geq T \\
\pi_{t}^{(n)} \times\left(1-\left[\frac{\Psi_{t-1}^{(n)}}{T}\right]\right) & \text { if } & \psi_{t-1}^{(n)}<T
\end{array}\right)
$$

In (4), $T$ is a threshold value having the same unit as that used for motion and particle angles. Re-weighted values are normalized and the likelihood probabilities of those particles that are moving in the same or similar direction to the target are increased.

\section{Proposed Method}

The decision of $T$ affects the performance of the method in (4) [14]. If $T$ is too narrow, tracking failure may occur due to less number of particles for state estimation In contrast, if there is no risk of failure, using a large number of particles is a computation load. These two problems are the main motivation for this study. A predefined $T$ value was used. In case of $\pi / 8$, the number of particles was minimum but tracker lost the target in some cases. When $T=\pi / 2$, best accuracy was observed with highest number of particles. So, we implemented an online thresholding adaptation scheme to prevent failures and usage of excessive particles. This scheme observes the particle amount and adapts $T$ according to the conditions given in fig.1. Initial and maximum value of $N$ is 100.

$$
\begin{gathered}
\text { if } 80 \leq N \leq 100 \text { OR } 25 \leq N \leq 40, T=\frac{\pi}{8} \\
\text { if } 0<N<25 \text { OR } 70 \leq N<80, T=\frac{\pi}{2} \\
\text { else } T=\frac{\pi}{4}
\end{gathered}
$$

Fig.1. Conditions of threshold adaptation.

According to the conditions in fig.1, threshold is assigned to $\pi / 8$, when the number of particles is in range of $[80,100]$ or $[25,40]$. The number of particle within the first range is excessive. There does not exist such a risk of failure within second range. Therefore, this adaptation prevents the excessive particle use and continue tracking in safe zone.

Threshold is assigned to $\pi / 2$, when the number of particles is in range of $(0,25)$ or $[70,80)$. First range is risky for tracking failures since there are only few particles for state estimation. This case occurs when the vehicle is turning or occluded. That's why particle number must increase not to miss the target. In the second range, tracking is performed 
accurately with sufficient number of particles. This adaptation prevents the tracking failures and keeps tracking with midlevel number of particles. In the rest, the tracker, keeps its value at $\pi / 4$ with sufficient amount of particles and accuracy. Range values are decided heuristically.

\section{Experiments and Results}

Experiments were conducted on two different traffic videos. First video is taken from a camera that is located on the road side of a bridge in Rep. of Korea. It contains high motion blur due to vibration of the bridge. The second video is taken from a public dataset [30]. In each video, horizontal and vertical distances between camera and road are different. They contain illumination variations, scale variations, occlusions, motion blur, in-plane rotation and background clutter.

Benchmarking of the proposed algorithm was conducted against condensation and Camshift algorithms. Results were analyzed in qualitative and quantitative measures.

\section{A. Evaluation Methods}

In this study, we use precision rate for quantitative analysis. A commonly used evaluation metric for tracking precision is the center location error. It is defined as the average Euclidean distance between the center location of the tracked targets and the actual ground truth. The average center location error over all the frames of the tracking sequence is used to express the overall performance for that tracking. However, in case of tracking failure, the tracker may move to random locations. This movement will increase the average center error. Thus, the measurement will not reflect the real performance of the tracker [31]. Precision plot shows the percentage of frames whose center error is less than a threshold. Precision score is for a tracker is represented by its score at threshold of 20 pixels [31].

One-pass evaluation (OPE) is used for robustness evaluation. It is the conventional way to evaluate a tracker throughout a test sequence with initialization from the ground truth in the first frame that the target appears.

\section{B. Qualitative Results}

In both videos, target vehicles are represented with color histograms in HSV domain. Same parameters for feature extraction are used for all three algorithms.

Fig.2 shows the tracking captures for first video. This test was made on a low-resolution video that contained background clutter, scale variation, in-plane rotation and motion blur due to vibration of camera. Target is the grey color car on the right lane. In addition to the noise effects mentined above, the color of the car is very similar to the road. The frames in column (a), (b) and (c) shows the trackers of the proposed, condensation and camshift algorithms respectively. All three algorithms can track the car until it is occluded by the poles. After occlusion, camshift tracker fails and condensation tracker misses the car while the proposed tracker continues tracking even the car gets occluded and reappears.

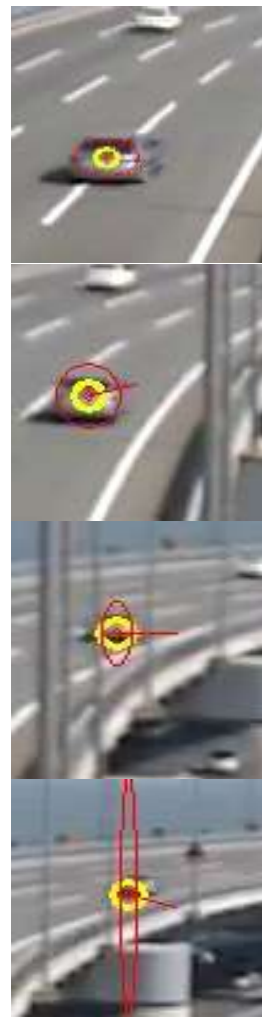

(a)

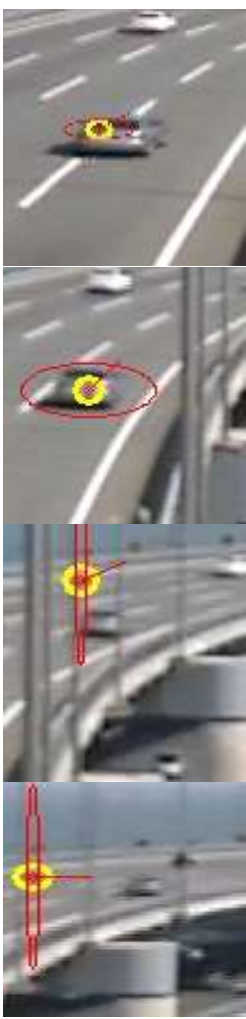

(b)

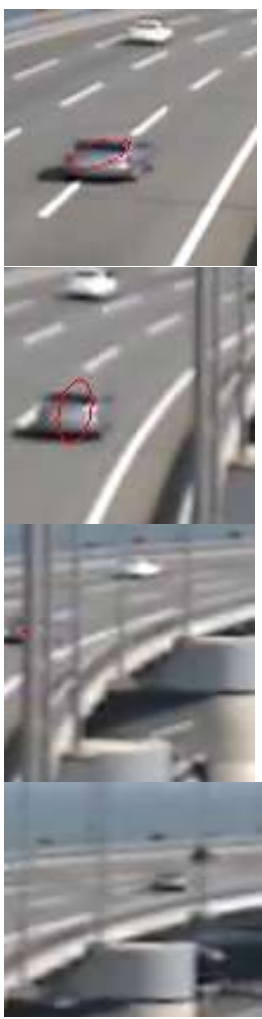

(c)
Figure 2. Tracking captures of first video: (a) Proposed tracker; (b) Condensation tracker; (c) Camshift tracker.

Fig.3 shows the tracking captures for the second video.This video is taken from a public dataset. It contains scale variation and in-plane rotation. Target blue car enters the frame from right bottom corner. Initially, there are several colors in its histogram.
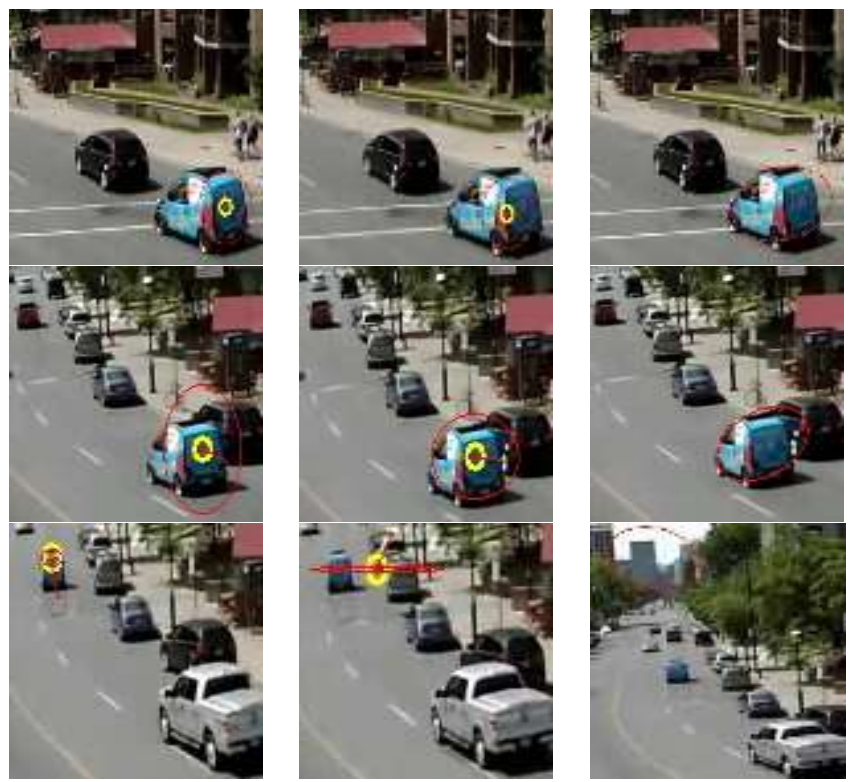


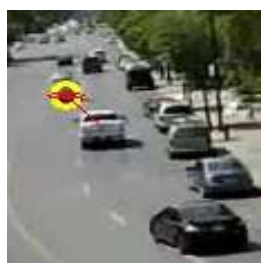

(a)

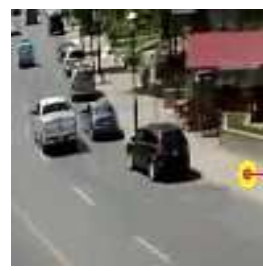

(b)

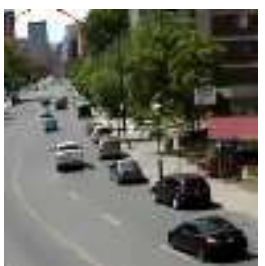

(c)
Figure 3. Tracking captures of second video: (a) Proposed tracker; (b) Condensation tracker; (c) Camshift tracker.

In the $3^{\text {rd }}$ frame, size of the car becomes relatively small compared to its initial size and looks like it only has blue color on it. At this frame, camshift and condensation trackers fail to track but proposed tracker keeps on tracking until the car becomes a very small point.

\section{Quantitative Results}

In this section, quantitative results of the two tests are given. Fig.4 shows the precision plot of first video with OPE evaluation. Scores written near each tracker are the precision scores at 20 pixels. Proposed PF has the highest score among three algorithms with 0.98 . It means that in $98 \%$ of frames of tracking, Euclidean distance between tracker and ground truth was less than 20 pixels.

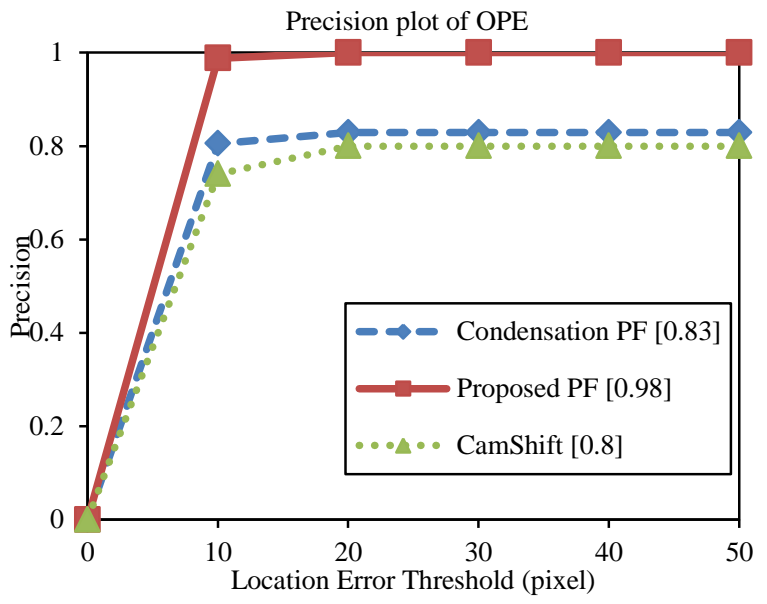

Figure 4. Precision plot for the first video.

Fig.5 shows the precision plot of the second video. Proposed tracker has the highest precision score. Although, both the camshift and condensation trackers miss the target at almost same location, precision score of camshift is much higher than the other. This is because, during tracking, camshift was more stable on the target.

Table I shows the average center location error of three trakcers for two videos. Condensation and camshift trackers have large error. The reason of it is that this calculation is done for the duration that vehicle was tracked by at least one tracker.

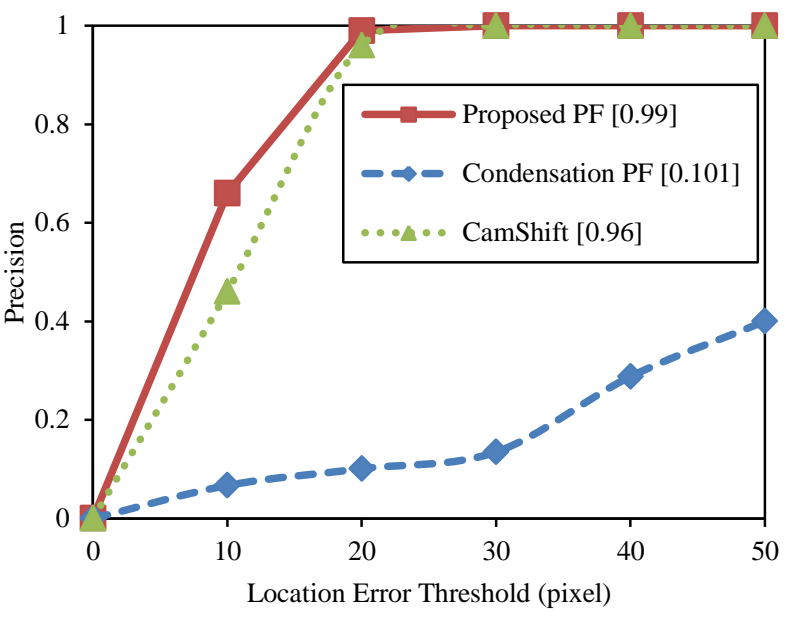

Figure 5. Precision plot for the second video.

While the proposed tracker continues tracking, the other trackers already fails. Thus their average error is much higher than the proposed tracker's error.

TABLE I. AVERAGE CENTER LOCATION ERROR (pixel)

\begin{tabular}{|c|c|c|c|}
\hline Video Clip & Proposed PF & Condensation & Camshift \\
\hline First Video & 4 & 82 & 96 \\
\hline Second Video & 9 & 200 & 220 \\
\hline
\end{tabular}

In addition to increase the accuracy of tracking, this method decreases the number of used particles for state estimation. This effect is shown in Table II. Proposed scheme, decreases the particle number by $40-50 \%$ on average.

TABLE II. AVERAGE NUMBER OF PARTICLES

\begin{tabular}{|c|c|c|}
\hline Video Clip & Proposed PF & Condensation \\
\hline First Video & 63 & 100 \\
\hline Second Video & 52 & 100 \\
\hline
\end{tabular}

\section{v. Conclusion}

This paper presents a novel vehicle tracking algorithm that filters and redistributes particles depending on their angular difference to the direction of the target vehicle. Particles having angular distance greater than the threshold are eliminated. The threshold is assigned online while the algorithm is running. Experiments were conducted on two videos with different characteristics and the results were compared with condensation and Camshift methods. The findings proved that proposed method improved the accuracy, reduced the particle consumption, and computational burden. 
Our method has become more stable and robust against occlusion, and noises.

\section{References}

[1] K. D. Sharma, A. Chatterjee, A. Rakshit, "A PSO - Lyapunov hybrid stable adaptive fuzzy tracking control approach for vision-based robot navigation," IEEE Trans. on Instr. and Meas., vol. 61, no. 7, pp. $1908-$ 1914, 2012

[2] Z.L. Szpak, J.R. Tapamo, "Maritime surveillance: Tracking ships inside a dynamic background using a fast level-set," Expert Syst. with App., vol. 38 , no. 6, pp. $6669-6680,2011$.

[3] K. Cannons, "A review of visual tracking," York Univ., Ontario, Canada, Tech. Rep. CSE - $2008-07,2008$.

[4] S. Sivaraman, M.M. Trivedi, "Looking at vehicles on the road: A survey of vision-based vehicle detection, tracking, and behavior analysis," IEEE Trans. on Intelligent Transp. Syst., vol. 14, no. 4, pp. 1773-1793, 2013.

[5] J. Gunnarsson, L. Svensson, L. Danielsson, F. Bengtsson, "Tracking vehicles using radar detections," in Proceedings of IEEE Intelligent Vehicles Symposium, vol. 1, pp. 269 - 302, Istanbul, Turkey, 2007.

[6] E. Galceran, E. Olson, R.M. Eustice, "Augmented vehicle tracking under occlusion for decision-making in autonomous driving," in Proc. of Int. Conf. Intelligent Robots and Systems, 2015, pp. 3559 - 3565, Hamburg, Germany, 2015

[7] J.C. McCall, M.M. Trivedi, "Video-based lane estimation and tracking for driver assistance: Survey, system and evaluation," IEEE Transactions on Intelligent Transportation Systems, vol.7, no.1, pp. 20 - 37, 2006.

[8] N.C. Mithun, N.U. Rashid, S.M.M. Rahman, "Detection and classification of vehicles from video using multiple time-spatial images," IEEE Trans. on Intelligent Transp. Sys., vol. 13, no. 3, pp. $1215-1225,2012$

[9] V.A. Prisacariu, I. Reid, "3D hand tracking for human computer interaction," Image and Vision Computing, vol. 30, no. 3, pp. 236 - 250, 2012.

[10] ] J. Hwang, K. Huh, "Vehicle detection system design based on stereo vision sensors," Int. J. of Automotive Tech., vol. 10, no. 3, pp. 373 $379,2009$.

[11] A. Sundaresan, R. Chellappa, "Multicamera tracking of articulated human motion using shape and motion cues," IEEE Trans. on Image Processing., vol. 18, no. 9, pp. 2114 - 2126, 2009.

[12] J. Arrospide, L. Salgado, "On-road visual vehicle tracking using markov chain monte carlo particle filtering with metropolis sampling," Int. J. of Automotive Tech., vol. 13, no. 6, pp. 955 - 961, 2012.

[13] W. Qi, X.Zhang, L. Chao, O.Yuanxin, S. Hao, "A robust approach for multiple vehicles tracking using layered particle filter," Int. J. of Electronics and Comm., vol. 65, pp. 609-618, 2011.

[14] M.E. Yıldırım, I.F. Ince, Y.B. Salman, J.K. Song, J.S. Park, B.W. Yoon, "Direction-based modified particle filter for vehicle tracking," ETRI Journal, vol. 38, no. 2, pp. 356 - 364, 2016

[15] X. Fei, K. Hashimoto, "An object-tracking algorithm based on particle filtering with region-based level set method," in Proc. of Int. Conf. on Intell. Robots and Syst., pp. 2908 - 2913, Taipei, Taiwan, 2010.

[16] Z. Kim, "Real-time object tracking based on dynamic feature grouping with background subtraction," in Proc. of IEEE Conf. of Com. Vis. and Pattern Recog., Anchorage, Alaska, pp. 1 - 8, 2008.

[17] A. Aksel, S.T. Acton, "Target tracking using the snake particle filter," in Proc. of IEEE Southwest Symposium of Image Anaysis and Interpretation, Austin, Texas, pp. 33 - 36, 2010.

[18] C.L. Dong and Y.N. Dong., "Survey on Video Based Vehicle Detection and Tracking Algorithms," J. of Nanjing University of Posts and Telecommunications: Nat. Sci., vol. 29, no. 2, pp. 88-94, 2009.

[19] Y.M. Chan, S.S., Huang, L.C. Fu, P.Y. Hsiao, "Vehicle detection and tracking under various lighting conditions using a particle filter," IET Intell. Transport Syst., vol. 6, pp. 1-8, 2012.

[20] Z. Fan, H. Ji, and Y. Zhang, "Iterative particle filter for visual tracking," Signal Processing: Image Comm., vol. 36, pp. 140-153, 2015.

[21] J.F. Dou, J.X. Li, "Robust Visual Tracking Based on Adaptively Multifeature Fusion and Particle Filter," Optik, vol. 125, no. 5, 2014, pp. 1680-1686, 2014.
[22] W. Li, P. Wang, H. Qiao, "Top-down visual attention integrated particle filter for robust object tracking," Signal Processing: Image Comm., vol. 43 , pp. $28-41,2016$.

[23] M.C. Ho, C.C. Chiang, Y.Y. Su, "Object tracking by exploiting adaptive region-wise linear subspace representations and adaptive templates in an iterative particle filter," Pattern Recog. Let., vol. 33, pp. 500 - 512, 2012.

[24] D. Comaniciu, V. Ramesh, P. Meer, "Kernel-based object tracking," IEEE Trans. on Pattern Analysis and Mach. Intell., vol. 25, pp. $564-$ $567,2003$.

[25] P. Perez, C. Hue, J. Vermaak, M. Gangnet, 'Color-based probabilistic tracking," in Proc. of $7^{\text {th }}$ European Conf. on Computer Vision, pp. 661 675, 2002.

[26] [26] W. Sun, X.C. Li, J.H. Qiu, F.S. Wang, "Iterated unscented kalman particle filter for visual tracking," Journal of Computer Information Systems, vol. 10, no.2, pp. $681-689,2014$.

[27] S.K. Zhou, R. Chellappa, and B. Moghaddam, "Visual Tracking and Recognition Using Appearance-Adaptive Models in Particle Filters," IEEE Trans. Image Process., vol. 13, no. 11, Nov., pp. 1491-1506, 2004

[28] K. Nummiaro, E. Koller-Meier and L.J.V. Gool, "An adaptive colorbased particle filter", Image Vision Comput., vol. 21, no. 1, pp. 99 $110,2003$.

[29] S.K. Zhou, R. Chellappa, and B. Moghaddam, "Visual Tracking and Recognition Using Appearance-Adaptive Models in Particle Filters," IEEE Trans. Image Process., vol. 13, no. 11, Nov., pp. 1491-1506, 2004.

[30] N. Saunier, H. Ardo, J.-P. Jodoin, A. Laureshyn, M. Nilsson, A. Svensson, L. Fernando Miranda-Moreno, G.-A. Bilodeau, and K. Astrom. Public video data set for road transportation applications. In Transportation Research Board Annual Meeting Compendium of Papers, 14-2379, 2014.

[31] B. Babenko, M.-H. Yang, and S. Belongie. Robust Object Tracking with Online Multiple Instance Learning, PAMI, vol.33, no. 7, pp. 1619-1632, 2011

\section{About Author (s):}

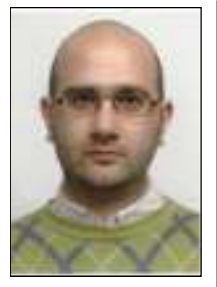

M. E. Y1ldırım received his $\mathrm{PhD}$ degree in electronics engineering, from the Graduate School of Electrical and Electronic Engineering, Kyungsung University, Pusan, Rep. of Korea, in 2014. He is currently lecturer in Dept. of EE at Bahcesehir University. His research area include image processing, computer vision, recognition and object tracking.

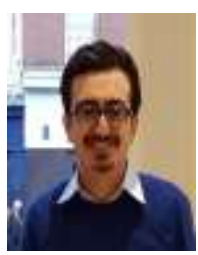

Burak Merdenyan is a $\mathrm{PhD}$ student at Computer Science Department of University of York, York. His research interest includes User Interface Design and Password Behaviour.

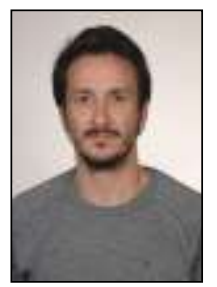

Y. Batu Salman received his $\mathrm{PhD}$ in IT Convergence Design from Kyungsung University, Pusan, Rep. of Korea, in 2010. $\mathrm{He}$ is currently vice director of Graduate School of Natural and Applied Sciences at Bahcesehir University. His research interests include human computer interaction, mobile programming and computer vision. 